\title{
Photometric Follow-up of the CoRoT Mission
}

\author{
The CoRoT Photometric Follow-Up Team ${ }^{1}$
}

email: hdeeg@iac.es

\begin{abstract}
A short overview of the motivation and techniques of the ground-based photometric follow-up program of the CoRoT mission is given.
\end{abstract}

\section{Overview}

Ground-based photometric follow-up plays an important role in the characterization of planet candidates found by the CoRoT spacecraft. Its principal raison d'être arises from the much higher spatial resolution of common ground-based telescopes in comparison to CoRoT's cameras. This allows the recognition of many transit candidates as being caused by contaminating eclipsing binaries. For the ground observations, 'on'-'off' photometry is now largely employed, in which only short time series during a transit and outside of a transit are observed and compared photometrically. During the first year of CoRoT's operation, the follow-up has been performed by a dedicated team with access to telescopes with sizes ranging from 0.7 to $8 \mathrm{~m}$. Experiences and techniques from this work may also be of interest to other transit-detection experiments, when the discovery instrument obtains data with a relatively low spatial resolution.

\section{The need for photometric follow-up}

CoRoT observes stellar fields with a fairly large psf, 20x6 and photometry is obtained in optimized apertures of corresponding large sizes. A faint transit signal detected by CoRoT may therefore arise from either the target or from another nearby source whose PSF falls into CoRoT's aperture - typically eclipsing binaries in the background. Groundbased follow-up photometry is able to resolve nearby stars at much higher resolution than CoRoT. Observing CoRoT-candidates at predicted transit times, the task of follow-up photometry is the identification of false alarms from any resolvable contaminating source.

CoRoTs photometric apertures have shapes that are adapted to the stellar contamination around a target, and contaminating stars satisfy one or both of the following conditions: They are are significantly fainter than the target, with fluxes $F_{c} \ll F_{t}$; and/or only a small fraction $k$ of their psf falls within the aperture. Hence a contaminant's expected 'false-alarm' flux variation, given by

$$
\frac{\Delta F_{c}}{F_{c}} \approx\left(\frac{F_{t}}{F_{c}}\right) k^{-1}(\Delta F / F)_{s}
$$

is typically much larger than CoRoTs observed flux variation $\Delta F_{s} / F_{s}$. This makes ground-based follow-up even of very shallow transit candidates possible, where the

${ }^{1}$ H. J. Deeg, S. Aigrain, A. Alapini, R. Alonso, J. M. Almenara, M. Barbieri, F. Bouchy, R. Dvorak, J. Eislffel, A. Erikson, M. Fridlund, M. Gillon, P. Eigmller, A. Hatzes, P. Kabath, T. Mazeh, C. Moutou, D. Queloz, H. Rauer, D. Rouan, B. Stecklum, M. Rabus, A. Shporer, B. Tingley, R. Titz 
ground-based reproduction of a transit on the main CoRoT target cannot be expected. The outcome of ground-based follow-up may then be one of the following cases:

- The transit is reproduced on main target $\Rightarrow$ candidate verified

- An eclipse is found on a nearby star $\Rightarrow$ false alarm

- No signal found on either target or nearby star $\Rightarrow$ verifies the transit candidate IF: - An on-target transit is too shallow for ground-based detection AND

- It can be demonstrated that none of the nearby stars has a signal strong enough to be a false alarm.

Since many CoRoT candidates have small amplitudes, typical outcomes of follow-up photometric observations are cases ii) or iii); that is, it is enough to demonstrate the presence of absence of relevant brightness variations in any of the nearby stars.

\section{Observing technique}

To perform the follow-up of large numbers of shallow candidates, the on-off observing technique has been developped, optimized to distinguish above cases ii) or iii): Instead of observing entire or partial transits, only short on and off-transit sections are observed and compared; acquired typically on different nights. The advantages are:

- A significant saving of observing time: $\approx 1 / 2 \mathrm{~h}$ on-transit is enough.

- Better performance with long transits if on and off data are acquired at similar extinction.

For the extraction of photometry, DECPHOT (Gillon et al. 2007) and VAPHOT (Deeg \& Doyle 2001) are mostly employed. For the latter, a special module to evaluate on-off differences has been developed, that includes an estimation of the nearby stars' false-alarm flux variation $\Delta F_{c} / F_{c}$ and contamination factors $k$, obtained from folding a simulated CoRoT PSF of the nearby stars over the target aperture.

\section{The CoRoT Photometric Follow-up Working Group}

To perform the observations and their analysis, the CoRoT Photometric Follow-up Working Group was formed which systematically reobserves CoRoT transit candidates from instruments available to them; with the bulk of observations being done with 1mclass telescopes and the occasional use of larger instruments for more difficult cases (faint targets, close contaminants, very shallow transits). To date, 32 candidates have been analyzed, of which 20 were on target (including the 5 detected planets) and 12 were false alarms on contaminants; all of them in the range $\Delta F_{s} / F_{s}$ of 0.1 to $1 \%$. A more detailed account of the motivation and techniques of the CoRoT photometric follow-up is the subject of a forthcoming dedicated publication.

\section{References}

Deeg, H. J. \& Doyle, L. R. 2001, in Third Workshop on Photometry, p. 85, ed. W. J. Borucki \& L. E. Lasher, 85

Gillon, M., Magain, P., \& Chantry, V., et al. 2007, in Astronomical Society of the Pacific Conference Series, Vol. 366, Transiting Extrapolar Planets Workshop, ed. C. Afonso, D. Weldrake, \& T. Henning, 113 